\title{
A New Sliding Surface for Discrete Second Order Sliding Mode Control for Time Delay Systems with Time Varying Uncertainties
}

\author{
Abdennabi Nizar \\ National Engineering School of Gabes \\ University of Gabes \\ Street of Medenine 6029 \\ Gabes, Tunisia \\ Research Unit: Numerical \\ Control of Industrial Processes
}

\author{
Ahmed Said Nouri \\ National Engineering School of Gabes \\ University of Gabes \\ Street of Medenine 6029 \\ Gabes, Tunisia \\ Research Unit: Numerical \\ Control of Industrial Processes
}

\begin{abstract}
In this work, a discrete second order sliding mode control with a new sliding function for a linear uncertain system with state delay is proposed. The systems are assumed to have structured mismatched time varying uncertainties. Firstly, a new sliding function include a present and a past value of the state, called dynamic surface, is designed by means of linear matrix inequalities (LMI). Then, a robust discrete second order sliding mode controller with this new function is investigated to overcome the effect of time delay and uncertainties in the closed loop. A numerical example illustrates the effectiveness and the advantages of the proposed approach.
\end{abstract}

\section{Keywords}

Discrete Time Delay Systems, Mismatched Time varying Uncertainties, Dynamic Sliding Function, Second Order Sliding Mode Control

\section{INTRODUCTION}

Time delay is a direct consequence of finite capability of information processing and data transmission among various parts of systems cannot, in many cases, be neglected and ignored or even approximated. This delay can affect the state or/and input or/and output and these can be constant or time varying, known or unknown, deterministic or stochastic depending on the systems under consideration. Systems with time delay exists in various fields of engineering: communication networks, biological reactors, chemical processes,.... Hence, the control problem of these systems, has received considerable attention over the past years, and different design approaches have been proposed [1], [2], [3]. However, they are sensitive to model uncertainties. So controlling this kind of systems can be a challenging task, especially in the presence of mismatched time varying uncertainties, parameter variations and external disturbances.

In the few past decades, the Discrete Sliding Mode Control (DSMC) technique has been widely studied and applied to such systems [4], [5]. This technique, first proposed in the early eighties, is characterized by its robustness with respect to modeling uncertainties and external disturbances, which satisfy the matching condition. In addition, this design approach enjoys the advantage of the reduction of the systems order [6],[7]. Despite these advantages, the Discrete Sliding Mode Control (DSMC) still encounters many problems: experimental implementation of the traditional (first-order) DSMC method introduces some drawbacks such as chattering effect, limited flexibility for the designer with a sliding function and sensibility to the unmatched uncertainties. Another disadvantage is the necessity of the whole state in designing the DSMC controller.

Many researchers are interested in solving such problems. Fridman et al. [8] proposed a descriptor approach with a recent result on sliding mode control to obtain a more efficient sliding mode controller for uncertain systems with time varying delay. In order to reduce the effect of the uncertainties, an estimation based on one-step delayed uncertainty approximation is exploited in [4]. In [9], the authors suppose that the uncertainties have a slower motion that the sampling frequency and then approximated it to construct his controller. In [10], [11], a Lyapunov-Krasovskii techniques-based delay-dependent descriptor approach to stability and control of linear uncertain time delay systems is proposed and combined with the sliding mode control to deal with the mismatched time varying uncertainties. The design of sliding surface and reaching motion controller for discretetime systems with time varying state delay, unmatched uncertainties and matched bounded unknown nonlinear function were the subject of the work [12].

But the problem of the chattering phenomenon still exist with the first sliding mode controller using the classical surface for linear time delay systems with mismatched time varying parameter uncertainties. Mihoub et al proposed in [13], a fuzzy supervised discontinuous term to reduce the chattering phenomenon and the reaching phase duration. In [5], the authors provide a solution to successively eliminate chattering based on a second order version of sliding mode (2-DSMC). However, if the time delay is not taken into account when the SMC is designed, it may lead to unstable behaviors, or at least, result in chattering behaviors. In [14], the authors use a functional surface to design the sliding mode controller for continuous time delay systems in order to reduce the effect of 
the delay and the chattering phenomenon. This controller is developed by means of an LMI approach. In [15], a similar sliding function is derived in the case of Uncertain Neutral time delay systems.

The present work extends the results given in our previous paper [16]. The design of the discrete sliding function for a class of linear time delay systems subject to norm-bounded uncertainty is presented.

The main result of our paper is the design of a discrete second order sliding mode controller without taking into account the upper and the lower bound of the mismatched time varying uncertainty. A delay independent sufficient condition for an asymptotic stability of the uncertain state delay system is derived in terms of LMIs. Then we propose a stable dynamic surface, which include the present and the past values of the state, by means of those LMIs. When these LMIs are feasible, this dynamic sliding function is used to design a new discrete second order sliding mode control in order to resolve the problems caused by the classical 2-DSMC controller. Simulation results are compared with those obtained by the convectional 2-DSMC and those obtained by the discrete predictive sliding mode control (PSMC) [17], [18].

This paper is organized as follows. In section two, the problem is formulated. In section three, the design of the classic sliding function is presented. Then we present the discrete second order sliding mode control with this classical function. In the fourth section, the design of the dynamic sliding function is given. The simulation results of the 2DSMC with dynamic function on a discrete time delay systems with unmatched time varying uncertainties and a comparison with the classical function and the discrete predictive sliding mode control are given in the fifth section.

\section{STATEMENT OF PROBLEM}

In this section, we focus on discrete linear time-delay systems with mismatched time varying parameter uncertainties that can be represented, possibly, after a change of state coordinates and input, into following regular form [11], [12], [16] :

$$
\left\{\begin{array}{l}
z_{1}(k+1)=\left(A_{11}+\Delta A_{11}\right) z_{1}(k)+\left(A_{d 11}+\Delta A_{d 11}\right) z_{1}(k-h)+\left(A_{12}+\Delta A_{12}\right) z_{2}(k) \\
z_{2}(k+1)=\sum_{i=1}^{2}\left(A_{2 i}+\Delta A_{2 i}\right) z_{i}(k)+\sum_{i=1}^{2}\left(A_{d 2 i}+\Delta A_{d 2 i}\right) z_{d i}(k)+B u(k) \\
z(k)=\varphi(k) \quad ; \quad k \in\{-h,-h+1, \ldots, 0\}
\end{array}\right.
$$

with $z_{1}(k) \in R^{n-m}, z_{2}(k) \in R^{m}, z(k)=\left[z_{1}(k) ; z_{2}(k)\right]^{T}$ is the state vector. $u(k) \in R^{m}$ is the control.

$A_{11}, A_{12}, A_{21}, A_{22}, A_{d 11}, A_{d 12}, A_{d 21}, A_{d 22}$ and $B$ are matrices with appropriate dimension.

$\Delta A_{11}, \Delta A_{12}, \Delta A_{21}, \Delta A_{22}, \Delta A_{d 11}, \Delta A_{d 12}, \Delta A_{d 21}$ and $\Delta A_{d 22}$ are unknowns and variables matrices. $\mathrm{h}$ is the constant delay and $\varphi(k) \forall\{k \in-h,-h+1, \ldots, 0\}$ is a given initial condition sequence. For the convenience of proof and without loss of generality, we can take the following assumptions.

\section{Assumption 1:}

All system states are available.

\section{Assumption 2:}

The pair of matrices $\left(A_{11}+A_{d 11}, A_{12}\right)$ in the nominal system is controllable.

\section{Assumption 3:}

The time-varying parameter uncertainties are assumed to be norm-bounded and with the form [4], [9], [10], [11] :

$\left[\begin{array}{lll}\Delta A_{11} & \Delta A_{12} & \Delta A_{d 11}\end{array}\right]=\left[\begin{array}{lll}E_{1} & E_{2} & E_{3}\end{array}\right]$

where $\mathrm{D}, \mathrm{E}_{1}, \mathrm{E}_{2}$ and $\mathrm{E}_{3}$ are known constant matrices and $G(k)$ is an unknown matrix with appropriate dimension and norm-bounded as follow $G(k)^{T} G(k)<I$ with I is the identity matrix.

The following lemma will be employed to handle the time varying norm-bounded uncertainties in the system.

\section{Lemma 1 [10]:}

Given matrices $\Gamma, D, E$ and $R=R^{T}$,

$$
\Gamma+D G(k) E+E^{T} G(k)^{T} D^{T} \mathrm{p} 0
$$

for all $G(k)$ satisfying $G(k)^{T} G(k)<R$, if and only if there exists $\lambda>0$ such that

$$
\Gamma+\lambda D D^{T}+\lambda^{-1} E^{T} R E \mathrm{p} 0
$$

The objective of this paper is to design a discrete second order sliding mode controller such that the closed-loop system is asymptotically stable.

This controller is designed in two steps:

- The design of a dynamic sliding surface that ensures the asymptotic stability of the time delay system using LMIs techniques is presented.

- A new discrete second order sliding mode control with a dynamic surface is given.

\section{THE DISCRETE SECOND ORDER SLIDING MODE CONTROL OF UNCERTAIN TIME-DELAY SYSTEM}

In this section, the classical second order sliding mode control for a class of linear discrete state time delay systems will be presented.

\subsection{Design of classical Sliding Surface using LMIs}

Many authors are interested in the design of the sliding function using the LMIs: a delay dependent and independent conditions are given in [3], [8], [14], [15] for a continuous time delay systems and in [4], [9], [11], [12] for discrete time delay systems.

For the uncertain discrete-time delay system (1), the classical sliding function is defined by 


$$
S(k)=\bar{C} z(k)=\left[\begin{array}{ll}
C & I
\end{array}\right] z(k)=z_{2}(k)+C z_{1}(k)
$$

Where $\bar{C}=\left[\begin{array}{ll}C & I\end{array}\right]$ with $C \in R^{m \times(n-m)}$ and I is the identity matrix.

Since $\left(A_{11}+A_{d 11}, A_{12}\right)$ is controllable, we can find a $C \in R^{m \times(n-m)}$ such that $D_{1}=A_{11}-A_{12} C+A_{d 11}$ is Hurwitz matrix. In sliding mode we have

$$
S(k)=0 \Rightarrow z_{2}(k)=-C z_{1}(k)
$$

Replacing (4) into system (1), we have

$$
z_{1}(k+1)=\left(A_{11}-A_{12} C+\Delta A_{11}-\Delta A_{12} C\right) z_{1}(k)+\left(A_{d 11^{+}}+\Delta A_{d 11}\right) z_{1}(k-h)
$$

Then, sub-system (5) represents the discrete-time sliding motion of system (1).

The following theorem gives a sufficient condition under which the sliding motion is stable.

\section{Theorem 1}

Consider the reduced system (5). Given positive integer $h$. The system is asymptotically stable if there exists matrices

$P=P^{T}>0, Q=Q^{T}>0$, some scalar $\lambda>0, \mathrm{w}_{1}$ with appropriate dimension satisfying the following LMI [9], [10]:

$$
\left[\begin{array}{cccc}
W-L & * & * & * \\
0 & -L & * & * \\
A_{11} L+A_{12} w_{1} & A_{d 11} L & \bar{L} & * \\
E_{1} L+E_{2} w_{1} & E_{2} L & 0 & -\lambda I
\end{array}\right]<0
$$

Where

$$
\begin{aligned}
& \bullet \bar{L}=-L+\lambda D D^{T}, \\
& \bullet W=P^{-1} Q P^{-1}, \\
& \bullet L=P^{-1}, \\
& \bullet w_{1}=-C L .
\end{aligned}
$$

and $(*)$ denotes the transposed elements in the symmetric positions.

\subsection{Second order sliding mode control with classical sliding function}

In high-order sliding mode control, the purpose is to force the

state to move on the intersection of the sliding surface $S(k)$ and its derivatives [5], [13]. In the case of the discrete second order sliding mode control (2-DSMC), the following relation must be verified

$$
S(k)=S(k+1)=0
$$

For simplicity, system (1) can be rewritten as follows

$$
\left\{z(k+1)=(A+\Delta A) z(k)+\left(A_{d}+\Delta A_{d}\right) z(k-h)+B u(k)\right.
$$

Where $z \in R^{n}$ is the state system vector. $u \in R^{m}$ is the control input. $A, A_{d}, \Delta A, \Delta A_{d}$ and $B$ are matrices with appropriate dimensions. $\mathrm{h}$ is the constant delay and $\varphi(k)$ is a vector-valued initial state function.

The sliding function is defined by

$$
S(k)=\bar{C} z(k)
$$

where $\bar{C}=\left[\begin{array}{ll}C & I\end{array}\right]$ with $C \in R^{m \times(n-m)}$ and $\mathrm{I}$ is the identity matrix. $\bar{C}$ is a coefficients vector obtained from the solution of the LMI (6) in theorem (1).

To design the discrete second order sliding mode controller, we consider system (7) and a new sliding surface $\sigma(k)$ is defined by [5].

$$
\sigma(k)=S(k+1)+\beta S(k)
$$

with $\beta \in[0 ; 1[$ is positive constant that ensures the stability of the function $\sigma(k)$. The first derivative of the sliding function $S(k)$ is

$$
S(k+1)=\bar{C} z(k+1)
$$

Noting that (10) and the system (7), $S(k+1)$ can be expressed as

$$
S(k+1)=\bar{C}\left((A+\Delta A) z(k)+\left(A_{d}+\Delta A_{d}\right) z(k-h)+B u(k)\right)
$$

The equivalent control $u_{e q}(k)$ can be obtained by letting

$$
\sigma(k+1)=0 \text { and } \sigma(k)=0
$$

which leads to the equivalent control $u_{e q}(k)$ to be defined by

$$
u_{e q}(k)=(\bar{C} B)^{-1}\left[-\beta S(k)-\bar{C}(A+\Delta A) z(k)-\bar{C}\left(A_{d}+\Delta A_{d}\right) z(k-h)\right]
$$

Where $(\bar{C} B)^{-1}$ is assumed to be non-singular matrix. The presence of the uncertainties ( $\triangle A$ and $\Delta A_{d}$ ) in the control law (13) make it unrealizable in the practice [9]. So the controller, without taking in account of the uncertainties nor an estimation of it, is given by

$$
u_{e q}(k)=(\bar{C} B)^{-1}\left[-\beta S(k)-\bar{C} A z(k)-\bar{C} A_{d} z(k-h)\right]
$$

Because of the ignored uncertainties, the control law (14) cannot bring the state trajectory on the sliding surface. This task should be accomplished by the discontinuous term [13]. By analogy with the continuous-time case, the integral of the discontinuous term, approximated by a first order transformation, is applied to system (7)

$$
u_{\text {dis }}(k)=u_{\text {dis }}(k-1)-T_{e} M \operatorname{sign}(\sigma(k))
$$

The control at the instant $(k)$ is then

$$
\begin{gathered}
u(k)=u_{e q}(k)+u_{d i s}(k) \\
u(k)=(\bar{C} B)^{-1}\left[-\beta S(k)-\bar{C} A z(k)-\bar{C} A_{d} z(k-h)\right] \\
+u_{d i s}(k-1)-T_{e} M \operatorname{sign}(\sigma(k))
\end{gathered}
$$


Under the control law (17), one can verify the finite-time convergence of the system (7) to the sliding surface $(S(k))$.

\section{THE DISCRETE SECOND ORDER SLIDING MODE CONTROL WITH DYNAMIC SLIDING FUNCTION}

In this section, we give our main results on the design of the sliding of a dynamic sliding function in term of LMIs.

\subsection{Second order sliding mode control with classical sliding function}

The system's response character is directed by the sliding surface when the system is in the sliding phase. For time delay systems, one must take into account the presence of the delay in the closed loop control in order to stabilize such systems. In our work, the response character is decided by the sliding function which depends on the present and delay states. Hence, the first procedure is to carefully choose the coefficient of this Dynamic Sliding function.

We define the dynamic sliding function for uncertain discretetime system with state delay (1) as follows

$$
S(k)=\bar{C} z(k)+C_{h_{1}}(k-h)=\left[\begin{array}{ll}
C & I
\end{array}\right] z(k)+C_{h_{1}} z_{1}(k-h)
$$

Where $\bar{C}=\left[\begin{array}{ll}C & I\end{array}\right]$ with $C \in R^{m \times(n-m)}$, I is the identity matrix and $C_{h}$ is a vector with the appropriate dimension to be designed. Since $\left(A_{11}+A_{d 11}, A_{12}\right)$ is controllable, we can find a $C \in R^{m \times(n-m)}$ and $\quad C_{h} \in R^{m \times(n-m)}$ such that $D_{2}=A_{11}-A_{12} C+A_{d 11}-A_{12} C_{h}$ is Hurwitz matrix.

In sliding mode we have

$S(k)=0 \Rightarrow z_{2}(k)=-C z_{1}(k)-C h^{z_{1}}(k-h)$

Substituting (19) into the system (1), we have the following reduced system

$$
\begin{aligned}
z_{1}(k+1)= & \left(A_{11}-A_{12} C+\Delta A_{11}-\Delta A_{12} C\right) z_{1}(k)+ \\
& \left(A_{d 11}-A_{d 12} C_{h}+\Delta A_{d 11}-\Delta A_{d 12} C_{h}\right) z_{1}(k)
\end{aligned}
$$

Then, the sub-system (20) represents the discrete-time sliding motion of system (1).

\section{Theorem 2}

Consider the reduced system (20). Given positive integer $h$. The system is asymptotically stable if there exists matrices $P=P^{T}>0, \quad Q=Q^{T}>0$, some scalar $\lambda>0, \quad w_{1}$ and $w_{2}$ with appropriate dimension satisfying the following LMI:

$$
\left[\begin{array}{cccc}
W-L & * & * & * \\
0 & -L & * & * \\
A_{11} L+A_{12} w_{1} & A_{d 11} L+A_{12} w_{2} & \bar{L} & * \\
E_{1} L+E_{2} w_{1} & E_{3} L+E_{2} w_{2} & 0 & -\lambda I
\end{array}\right]<0
$$

where

$$
\begin{aligned}
& \bullet \bar{L}=-L+\lambda D D^{T}, \\
& \bullet W=P^{-1} Q P^{-1}, \\
& \bullet L=P^{-1}, \\
& \bullet w_{1}=-C L, \\
& \bullet w_{2}=-C_{h} L
\end{aligned}
$$

Given this theorem, one can fix the coefficients of this surface optimally based on stability analysis with the candidate Lyapunov function.

\section{Proof}

Consider a candidate Lyapunov function as follows:

$$
V(k)=z_{1}^{T}(k) P z_{1}(k)+\sum_{\alpha=k-h}^{k-1} z_{1}^{T}(\alpha) Q z_{1}(\alpha)
$$

with $P$ and $Q$ are a symmetric definite positive matrices. The first order difference equation of the Lyapunov function is

$$
V(k+1)=z_{1}^{T}(k+1) P z_{1}(k+1)+\sum_{\alpha=k-h+1}^{k} z_{1}^{T}(\alpha) Q z_{1}(\alpha)
$$

The inequality $\Delta V(k)=V(k+1)-V(k)$ is known to show that the trajectory of system (5) can be driven onto the sliding mode plane in finite time, then

$$
\begin{aligned}
& \Delta V(k)=V(k+1)-V(k) \\
& =z_{1}^{T}(k+1) P z_{1}(k+1)+\sum_{\alpha=k-h+1}^{k} z_{1}^{T}(\alpha) Q z_{1}(\alpha) \\
& -z_{1}^{T}(k) P z_{1}(k)+\sum_{\alpha=k-h}^{k-1} z_{1}^{T}(\alpha) Q z_{1}(\alpha) \\
& =\underline{A}(k)\left(\left[\begin{array}{ll}
\bar{A}_{1} & \bar{A}_{d 1}
\end{array}\right]^{T} P\left[\begin{array}{ll}
\bar{A}_{1} & \bar{A}_{d 1}
\end{array}\right]^{+S}\right) \operatorname{2q}(k) \\
& Z_{p}(k)=\left[\begin{array}{c}
z_{1}(k) \\
z_{1}(k-h)
\end{array}\right] \quad ; \quad S=\left[\begin{array}{cc}
Q-P & 0 \\
0 & -Q
\end{array}\right] \\
& \bar{A}_{1}=A_{11}-A_{12} C \text { et } \quad \bar{A}_{d 1}=A_{d 11}-A_{12} C_{h}
\end{aligned}
$$

and the dynamic (5) is asymptotically stable if and only if

$$
\left(\left[\begin{array}{ll}
\bar{A}_{1} & \bar{A}_{d 1}
\end{array}\right]^{T} P\left[\begin{array}{ll}
\bar{A}_{1} & \bar{A}_{d 1}
\end{array}\right]+S\right)<0
$$

Using the Schur Complement, the inequality (22) can be written as

$$
\left[\begin{array}{ccc}
Q-P & * & * \\
0 & -Q & * \\
\bar{A}_{1} & \bar{A}_{d 1} & -P^{-1}
\end{array}\right]<0
$$

with $(*)$ denote the transpose of the element in the symmetric positions.

We can write:

$$
\left[\begin{array}{ccc}
Q-P & * & * \\
0 & -Q & * \\
A_{11}-A_{12} C & A_{d 11^{-}-A_{12} C_{h}} & -P^{-1}
\end{array}\right]<0
$$


To get the (23) in LMIs form, we consider the congruence transformation $\operatorname{diag}\left\{P^{-1}, P^{-1}, \mathrm{I}\right\}$ and we take, $L=P^{-1}$, $\bar{L}=-L+\lambda D D^{T}, W=P^{-1} Q P^{-1}, L=P^{-1}, w_{1}=-C L$ and $w_{2}=-C_{h} L$ then the inequality (23) can be written in the following LMI

$$
\left[\begin{array}{cccc}
W-L & * & * & * \\
0 & -L & * & * \\
A_{11} L+A_{12} w_{1} & A_{d 11} L+A_{12} w_{2} & \bar{L} & * \\
E_{1} L+E_{2} w_{1} & E_{3} L+E_{2} w_{2} & 0 & -\lambda I
\end{array}\right]<0
$$

Then Theorem 2 is verified.

\subsection{Second order sliding mode control with dynamic surface}

For the system (7), the dynamic sliding surface is defined by the following equation

$$
S(k)=\bar{C} z(k)+C_{h} z(k-h)
$$

where $\bar{C}$ and $C_{h}$ are vectors with the appropriate dimensions obtained from the solution of the LMI (21) in theorem (2).

After choosing optimally the coefficients of the sliding function, we design the discrete second order sliding mode controller, by analogy with the classical case. Now we consider a new system, with the sliding functions $S(k)$ and $S(k+1)$ given by

$$
\left\{\begin{array}{l}
S(k)=\bar{C} z(k)+C_{h} z(k-h) \\
S(k+1)=\bar{C} z(k+1)+C_{h} z(k-h+1)
\end{array}\right.
$$

The sliding function of this new system $\sigma(k)$ is taken in the following linear form:

$$
\sigma(k)=S(k+1)+\beta S(k)
$$

with $\beta \in[0 ; 1[$ a constant coefficient that guarantees the convergence of $\sigma(k)$ to zero.

From the sliding surface (24) we deduce

$$
S(k+1)=\bar{C} z(k+1)+C_{h} z(k-h+1)
$$

Replacing $z(k+1)$ from (7), we have

$$
\begin{aligned}
S(k+1)= & \bar{C}\left((A+\Delta A) z(k)+\left(A_{d}+\Delta A_{d}\right) z(k-h)+B u(k)\right) \\
& +C_{h} z(k-h+1)
\end{aligned}
$$

The equivalent control that forces the system to evolve on the sliding surface, noted $u_{e q}(k)$, is deduced from

$$
\sigma(k+1)=0 \text { and } \sigma(k)=0
$$

Equations (27) and (28) give

$$
\sigma(k+1)=S(k+1)+\beta S(k)
$$

and

$$
\begin{aligned}
S(k+1)= & \sigma(k+1)-\beta S(k)=\bar{C} z(k+1)+C z(k-h+1) \\
= & \bar{C}\left((A+\Delta A) z(k)+\left(A_{d}+\Delta A_{d}\right) z(k-h)+B u_{e q}(k)\right) \\
& +C_{h} z(k-h+1)
\end{aligned}
$$

By ignoring the uncertainties, the equivalent control is taken as follows:

$$
u_{e q}(k)=-(\bar{C} B)^{-1}\left[-\beta S(k)-\bar{C} A z(k)-\bar{C} A_{d} z(k-h)+C_{h} z(k-h+1)\right]
$$

The same switching control function $u_{d i s}(k)$ (15) is used (sign of the new sliding function $\sigma(k)$ ) in the following form:

$$
u_{d i s}(k)=u_{d i s}(k-1)-T_{e} M \operatorname{sign}(\sigma(k))
$$

Finally the control law applied to system (1) is

$$
u(k)=u_{e q}(k)+u_{d i s}(k)
$$

\subsection{Simulation Result}

Consider the uncertain discrete-time state delay system described by

$$
\left\{\begin{array}{l}
x(k+1)=(A+\Delta A) x(k)+\left(A_{d}+\Delta A_{d}\right) x(k-h)+B u(k) \\
x(k)=\varphi(k) \quad ; k \in\{-h,-h+1, \mathrm{~K}, 0\}
\end{array}\right.
$$

with

$$
A=\left[\begin{array}{cc}
1.2 & 0.1 \\
1 & 0.6
\end{array}\right] ; A_{d}=\left[\begin{array}{cc}
-0.2 & 0 \\
1 & -1
\end{array}\right] ; B=\left[\begin{array}{l}
0 \\
1
\end{array}\right]
$$

Where $\beta=0.1, M=0.005, h=10, T_{e}=0.01$ and the initial states of the system are $\phi(k)^{T}=\left[\begin{array}{ll}-0.7 & 0.5\end{array}\right]$ for $k \in[-10 ; 0]$. The vectors of the sliding functions (classic and dynamic) $\bar{C}$ and $C_{h}$ are designed according to theorem (1) and theorem (2). The mismatched time varying parameter uncertainties are

$$
\begin{gathered}
\Delta A=\left[\begin{array}{ll}
\Delta A_{11} & \Delta A_{12} \\
\Delta A_{21} & \Delta A_{22}
\end{array}\right]=\left[\begin{array}{ll}
0.04 \cos \left(20 k \pi T_{e}\right) & 0.04 \cos \left(20 k \pi T_{e}\right) \\
0.03 \sin \left(20 k \pi T_{e}\right) & 0.06 \sin \left(20 k \pi T_{e}\right)
\end{array}\right] ; \\
\Delta_{d}=\left[\begin{array}{ll}
\Delta A_{d 11} & \Delta A_{d 12} \\
\Delta A_{d 21} & \Delta A_{d 22}
\end{array}\right]=\left[\begin{array}{cc}
0.02 \cos \left(20 k \pi T_{e}\right) & 0.08 \cos \left(20 k \pi T_{e}\right) \\
0.09 \sin \left(20 k \pi T_{e}\right) & 0.09 \sin \left(20 k \pi T_{e}\right)
\end{array}\right]
\end{gathered}
$$

In order to compare the performances of the proposed second order sliding mode control with the dynamic sliding surface with those of the second order sliding mode control with classical surface and with the discrete predictive sliding mode control (PSMC) [17], we apply them in the same systems 
model (31). Using the Matlab LMI Control Toolbox, it can be obtained that LMIs (equations 6 and 27) in theorems (1) and (2) have feasible solutions and are given in table (1).

Table 1. Comparison of simulation results.

\begin{tabular}{|c|c|c|}
\hline 1 & Classic function & Dynamic function \\
\hline Best value of $\mathrm{t}(\mathrm{s})$ & 0.892318 & 3.3236 \\
\hline$\lambda$ & 19.8702 & 51.2867 \\
\hline $\mathrm{P}$ & 0.0127 & 0.0097 \\
\hline $\mathrm{Q}$ & 0.0086 & 0.0064 \\
\hline $\mathrm{w}_{1}$ & 62.5379 & 81.7638 \\
\hline $\mathrm{w}_{2}$ & - & -61.5277 \\
\hline
\end{tabular}

Then, using the explicit formula, the sliding functions are obtained as follows:

- Classic function:

$$
S(k+1)=-0.7959 x_{1}(k)+x_{2}(k)
$$

- Dynamic function

$$
S(k+1)=-0.7971 x_{1}(k)+x_{2}(k)+0.5998 x_{1}(k-h)
$$

Comparisons between system state trajectories for the three algorithms are illustrated in Fig1 and Fig2. Fig3 depicts the control input signal. The resulting sliding surface is presented in Fig4. It is observed from Fig1 and Fig2 that the system can be stabilized quickly by the proposed method and the reaching motion satisfies the sliding reaching condition in spite of the time-delays in state and the time-varying uncertainties without any oscillations around the sliding surface (Fig4).

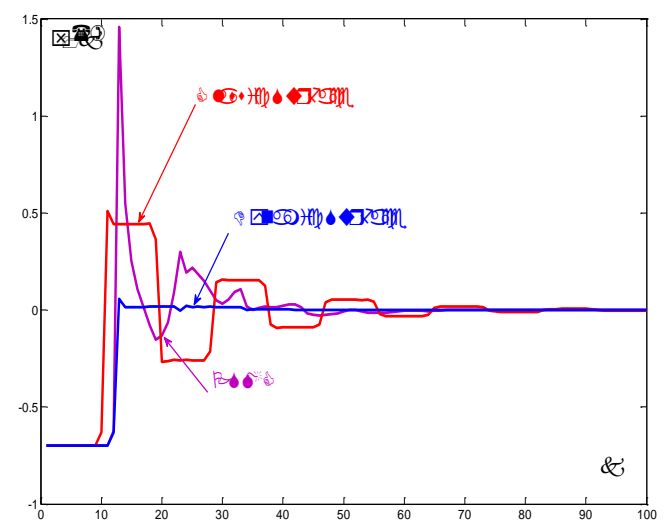

Fig 1: The second state space evolution.

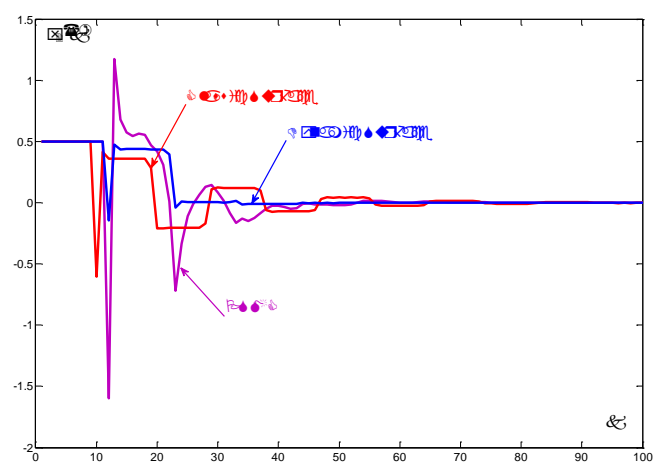

Fig 2: The second state space evolution.

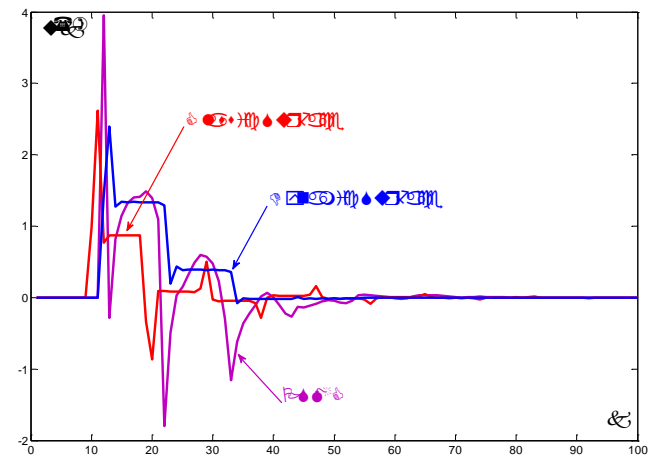

Fig 3: The control evolution.

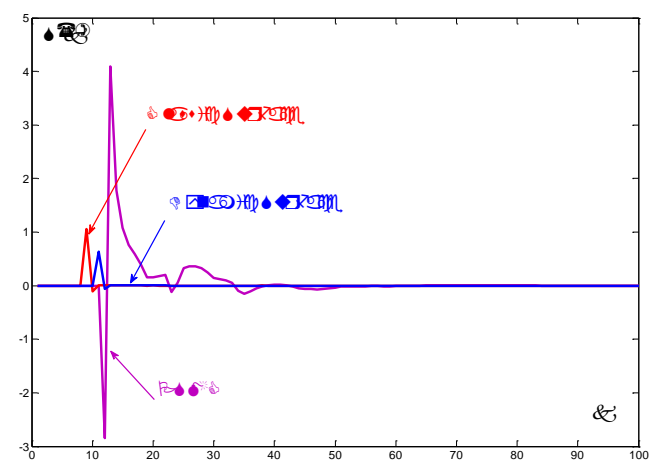

Fig 4 : The sliding function evolution.

Simulation results illustrate that the proposed approach in this paper is feasible and effective for linear discrete-time systems with state time-delay and time-varying uncertainties.

\section{CONCLUSION}

In this paper the problem of the stabilization of the discrete linear time delay systems subject to time-varying uncertainties has been investigated. Using the LMI solvers, a sufficient condition was given to guarantee the existence of a new stable sliding function. The proposed controller using this sliding function that include a present and delay state, ensures the asymptotic stability and the reduction of the chattering phenomenon. This controller presents a great advantage that it does not require parameter uncertainties estimation or a predictive sliding mode controller. In the case of a variable 
delay this study is not available. To ensure stability of the closed loop, we must use the Lyapunov theory in choosing an available Lyapunov function. This proposed study is under develloppement. Therefore, its implementation in real time is quite easy. Simulations indicate that this controller has a better performance than the one designed using the classical function. Simulation results illustrate that the proposed approach in this paper is feasible and effective for linear discrete-time systems with state time-delay and time-varying uncertainties.

\section{ACKNOWLEDGMENTS}

"This work was supported by the Ministry of the Higher Education and Scientific Research in Tunisia”.

\section{REFERENCES}

[1] Richard, J. P., 2003. Time-delay systems: an overview of some recent advances and open problems. Automatica, vol. 39, no. 5, pp. 1669-1694.

[2] Camacho, O., and Roujas, R. and Garcia-Gabin, W., 2007. Some long time delay sling mode control approaches, ISA. Transactions, vol. 46, pp. 95-101.

[3] Seuret, A., 2006. Commande et Observation des Systèmes à Retards Variables: Théorie et Applications, Thesis.

[4] Chang, Pai, M., 2010. Discrete-time sliding mode control for uncertain systems with state and input delays, International Journal of Systems Science, vol.12, pp. $1501-1510$.

[5] Mihoub. M., Nouri .A. S. and Ben Abdennour, R.,2009 Real-time application of discret second ordre silding mode control to a chemical reactor. Control Engineering Practice, vol.17, pp.1089-1095.

[6] Buhler, H. 1986.Réglage par mode de glissement, Lausanne Presse Polytechnique Romandes Lausanne, 1986.

[7] Lopez .P. and Nouri. A. S, 2006. Théorie élémentaire et pratique de la commande par les régimes glissants. Mathématiques et applications 55, SMAI, Springer Verlag.

[8] Fridman, E., Gouaisbaut, F., Dambrine, M., and Richard J. P, 2003. Sliding mode control of systems with time- varying delays via descriptor approach, International Journal of Systems Science, vol.34, pp. 553-559.

[9] Minxiu, Y., Yuanwei, J., Youguo, H., 2008. The Output Feedback Variable Structure Control for Discrete-Time Systems with Uncertainties and Time Delay, International Conference on Intelligent Computation Technology and Automation, pp.516-520.

[10] Yan, M. and Shi, Y., 2008. Robust sliding-mode control of uncertain discrete time systems with time delay, CIET Control Theory Appl, vol. 2, no. 8, pp. 662-674.

[11] Huimin, W. C. and Gao, C., 2010. Discrete SlidingMode Control of Uncertain Systems with Time Delays, Proccedings of the International Conferenceon Modelling, Identification and Control, Okyama, Japan, pp.882-885.

[12] Yan . M., Mehr .A. S., and Shi, Y, 2008. Discrete-time sliding-mode control of uncertain systems with timevarying delays via descriptor approach. Control Science and Engineering.

[13] Mihoub . M., Messaoud. A, Lateif. M, Nouri , A. S. and Ben. Abdennour, R., 2008, Fuzzy discontinuous term for a second order asymptotic DSMC: An experimental validation on a chemical reactor". Asian Journal of Control, Vol.00, pp.1-11.

[14] Richard , J. P., Gouaisbaut, F. and Perruquetti, W., 2001 Sliding mode control in the presence of delay. KYBERNETIKA, vol.37, pp. 277-294.

[15] Youguoa, H., zhaoleib and Muyongb, Z., 2011. Sliding Mode Controlfor a Class of Uncertain Neutral Delay Systems, Advanced in Control Engineering and Information Science, vol. 15, pp. 1181-1185.

[16] Abdennabi, N. and Nouri, A. S., 2012. A new sliding surface for discrete second order Sliding Mode Control of Time Delay Systems, 9th International MultiConference on Systems, Signals and Devices, Tunisia.

[17] Ben Mansour. H and N., Nouri, A. S., 2012. Discrete predictive sliding mode control of uncertain systems, 9th International Multi- Conference on Systems, Signals and Devices, Tunisia.

[18] Mao, H. W. and Shuhuan. L., 2009. Discrete sliding mode prediction control of uncertain switched systems, Journal of systems Engineering and Electronics, vol. 20, pp. 1065-1071. 